\title{
Medial collateral ligament injuries of the knee: current treatment concepts
}

\author{
Lan Chen · Paul D. Kim · Christopher S. Ahmad • \\ William N. Levine
}

Published online: 7 December 2007

(C) Humana Press 2007

\begin{abstract}
The medial collateral ligament is one of the most commonly injured ligaments of the knee. Most injuries result from a valgus force on the knee. The increased participation in football, ice hockey, and skiing has all contributed to the increased frequency of MCL injuries. Prophylactic knee bracing in contact sports may prevent injury; however, performance may suffer. The majority of patients who sustain an MCL injury will achieve their preinjury activity level with non-operative treatment alone; however, those with combined ligamentous injuries may require acute operative care. Accurate characterization of each aspect of the injury will help to determine the optimum treatment plan.
\end{abstract}

Keywords Medial collateral ligament - Knee joint . Humans - Joint instability $\cdot$ Sprains and strains

\section{Introduction}

The medial collateral ligament is one of the most commonly injured ligaments of the knee. Most injuries result from a valgus force on the knee from direct contact or with cutting maneuvers when an athlete plants his/her foot and then forcefully shifts directions [1]. The popularity of football, ice hockey, and skiing has all contributed to the increased frequency of MCL injuries. The role of bracing is controversial because it may help prevent injury but may also restrict performance.

L. Chen · P. D. Kim · C. S. Ahmad · W. N. Levine $(\square)$ Department of Orthopedic Surgery, Columbia University Medical Center, 622 West 168th Street, PH11-Center, New York, NY 10032, USA

e-mail:WNL1@columbia.edu
The treatment of medial-sided knee injuries has evolved from aggressive surgical treatments to mostly non-operative management with surgery reserved for chronic MCL deficiency that failed non-operative treatment or more severe, complex injuries. The majority of athletes who sustain an MCL injury will achieve their pre-injury activity level with non-operative treatment; however, those with combined ligamentous injuries may require acute operative care. We will review the functional anatomy, clinical and radiographic evaluation, and treatment options for isolated MCL injuries and combined ligament injuries of the knee.

\section{Functional anatomy}

Static stabilizers of the medial knee include the superficial MCL (s-MCL), the deep MCL (d-MCL) or medial capsular ligament, and the posterior oblique ligament. Dynamic stabilizers include the musculotendonous units of the semimembranosus, quadriceps, and pes anserinus. Warren and Marshall first described the anatomy in three layers: superficial (I), intermediate (II), and deep (III) [2]. The superficial layer (I) consists of the deep crural fascia which invests the sartorius and quadriceps and continues into the deep fascia of the lower extremity to cover the gastrocnemius and popliteal fossa. Layer II (intermediate) comprises the s-MCL and medial patellofemoral ligament (MPFL). Layer III is the deep layer, including the joint capsule and d-MCL.

The s-MCL is a broad ligament that attaches at the medial femoral epicondyle and inserts just below the pes anserinus, $4-5 \mathrm{~cm}$ distal from the joint line (Fig. 1a). It is anterior fibers are taut during flexion and lax during full extension [3]. Just deep into the s-MCL, the d-MCL is a confluence of numerous soft tissue structures including the 


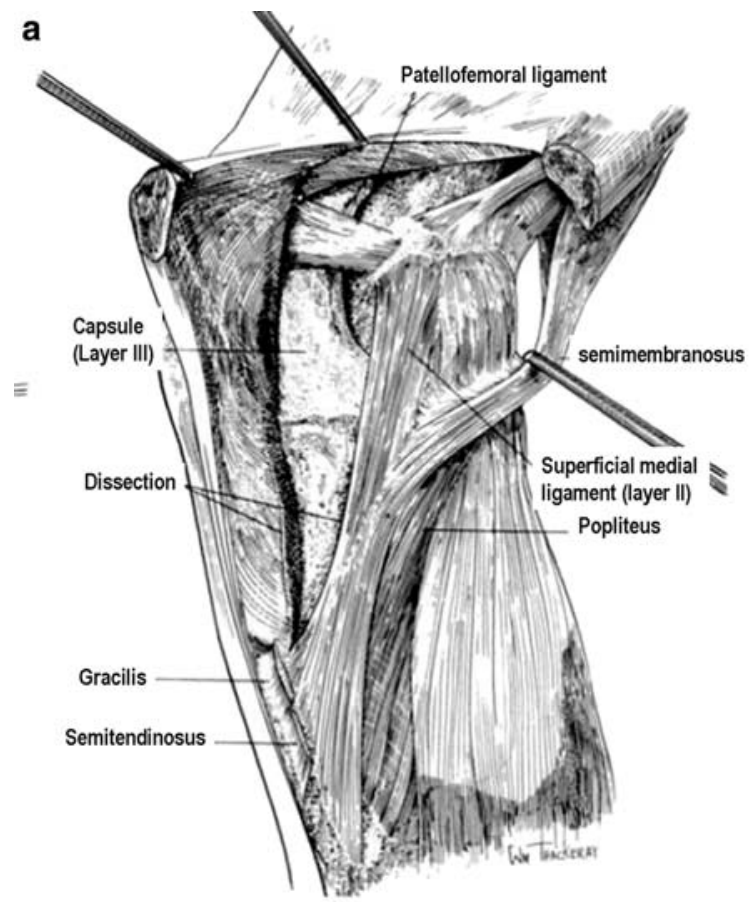

Fig. 1 (a) The superficial medial collateral ligament lies in the intermediate layer, layer II. The s-MCL is a broad structure that originates at the medial femoral epicondyle to insert $4-5 \mathrm{~cm}$ below the joint line. Anterior to this lies the capsule which is in the deep layer, layer III. (b) Deep into the s-MCL, the d-MCL is a confluence

meniscofemoral, meniscocapsular, and meniscotibial ligaments (Fig. 1b). A bursa separates the deep and superficial MCL and is biomechanically important by allowing anteroposterior excursion of the s-MCL during flexion and extension [1]. Posterior to the MCL is the posteriomedial corner, made up of a condensation of the capsule called the posterior oblique ligament (POL). This complex is tight in extension and becomes confluent with the posterior joint capsule.

The MCL is the primary restraint against valgus stress. At $25^{\circ}$ of flexion, the MCL provides $78 \%$ of the valgusrestraining force. In extension, the ACL and posteriomedial corner (POL, medial meniscus, and semimembranosus) also contribute to valgus stress and the MCL provides 57\% of the restraining force against valgus stress [4]. Cutting studies have shown a $3-5 \mathrm{~mm}$ joint opening in extension after the MCL is transected [3]. Additional transection of the PMC increases the joint opening. In general, an isolated MCL tear leads to valgus laxity in flexion, while additional injury to the secondary valgus restraints (PMC or ACL) leads to increased laxity in extension.

Gardiner et al. [3] found that the amount of strain to valgus stress over different areas of the MCL is highly dependent on the flexion angle of the knee. The highest strain was over the posterior part of the MCL at the femoral insertion when the knee is in full extension. Strain in this area

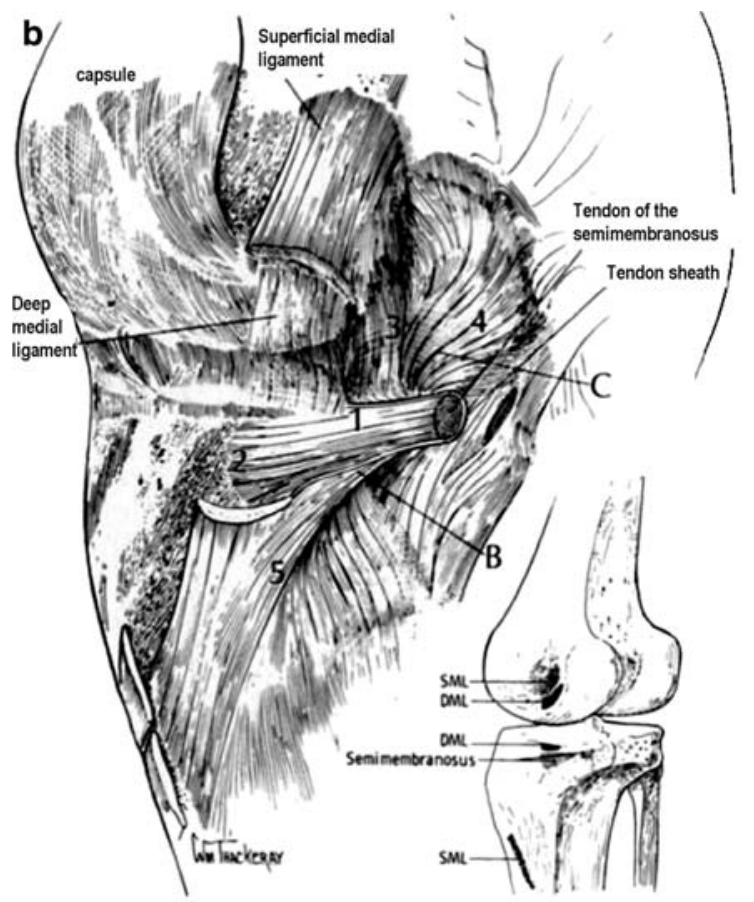

of numerous soft tissue structures including the meniscofemoral, meniscocapsular, and meniscotibial ligaments. Note that the semimembranosus tendon $(1,2)$ and tendon sheaths $(3,4,5, \mathrm{~B})$ are distal to the d-MCL. C represents the posterior oblique ligament. (From [2], with permission)

decreased with increasing flexion angles, while strain in the anterior fibers remained relatively constant throughout different flexion angles. This study correlates with several radiographic studies that indicate the posterior femoral attachment of the MCL is the most common location for MCL ligament injuries (Fig. 2).

\section{Clinical exam}

To obtain an accurate exam of ligament laxity, the patient should be relaxed and the contralateral leg is used as a control for any medial joint line opening. A gentle valgus force should be applied to the leg with the knee in $30^{\circ}$ of flexion thereby isolating the MCL (Fig. 3). In large patients, the thigh can rest on the table, while the lower leg is hung off the table with the foot and ankle supported. As shown in biomechanical studies, even a small medial joint opening of $5-8 \mathrm{~mm}$ is indicative of significant injury to the MCL [4].

In addition to assessing the MCL, other surrounding soft tissue injuries around the knee also need to be examined. The knee is placed into full extension and a valgus force is repeated. Increased medial joint space opening indicates additional posterior oblique ligament injury, ACL, or PCL injury. A more subtle examination skill is the evaluation of 


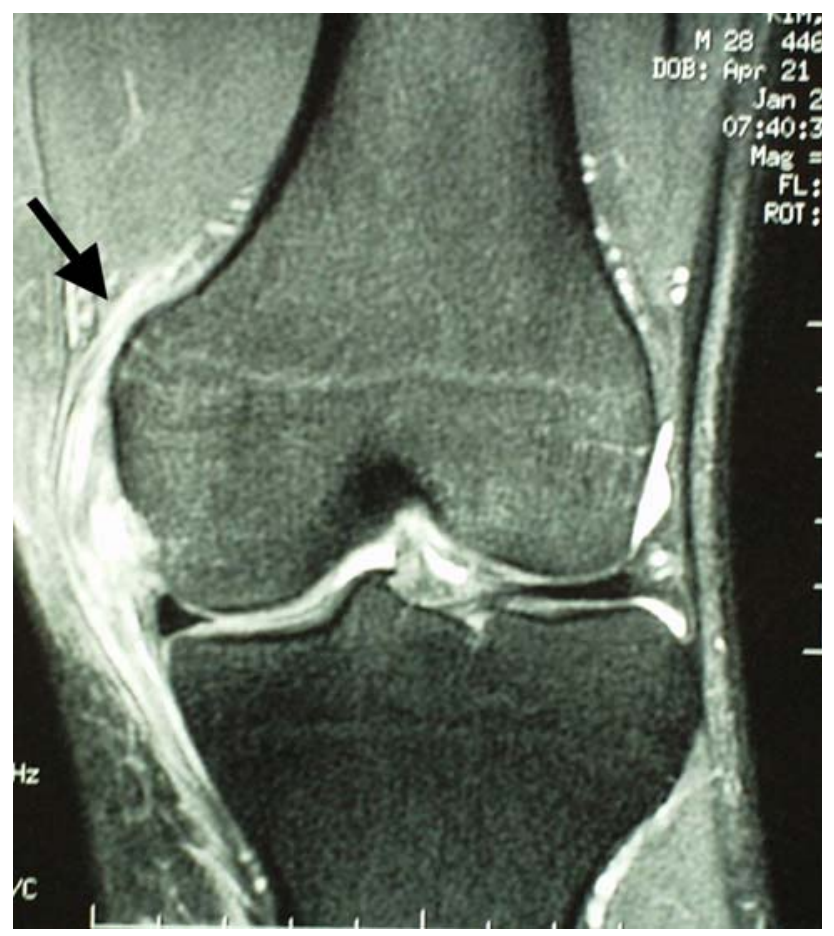

Fig. 2 Coronal MRI demonstrating complete grade 3 MCL tear from the femoral origin (black arrow)

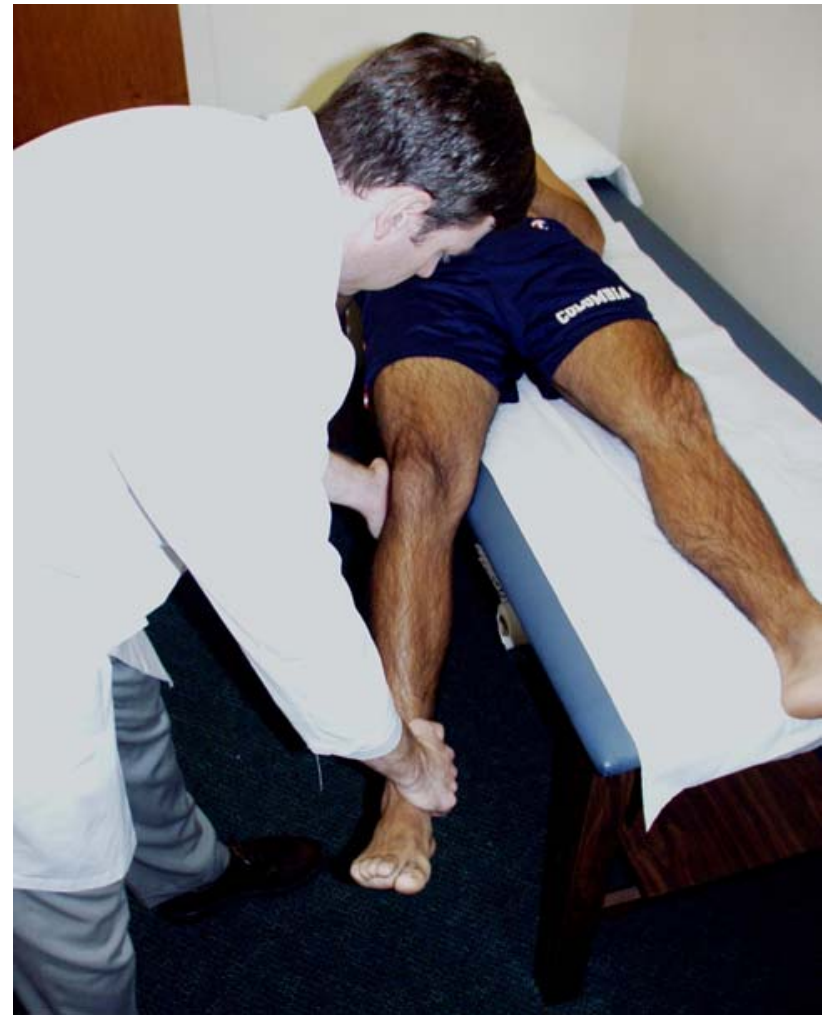

Fig. 3 Physical examination of a right knee-notice distal hand applying valgus force to foot the quality of the endpoint in medial joint opening. In isolated complete MCL tears, the soft end point during valgus stress with the knee in $30^{\circ}$ of flexion is the intact cruciate ligament. This is compared to the firm endpoint to valgus stress on the contralateral limb [1].

Other important information in the history and physical includes the location of pain and swelling, time and onset of swelling, sensation of a pop or tear, and presence of deformity immediately after injury [5]. The location of swelling is a clue to the extent of injury. Isolated MCL injuries often present as localized soft tissue swelling, whereas combined ACL/PCL tears result in significant hemarthrosis and generalized knee effusion. Hughston's series showed that the location of edema and tenderness accurately localized injury to the MCL in 64 and $76 \%$, respectively [6].

Frequently, the best time to perform an accurate exam is immediately after injury when muscle spasm have not occurred. This opportunity is only available to team physicians or trainers who are present at the sidelines. However, after pain, swelling, and muscle spasm has set in, even experienced clinicians can have difficulty performing an accurate exam [1].

Standard radiographic knee radiographs are indicated in suspected MCL injuries. An AP weight-bearing, lateral, and sunrise view should be obtained. Bony avulsions or osteochondral fragments can significantly change the treatment plan. Stress views are important in skeletally immature patients for evaluating physeal injuries [7]. Lateral tibial plateau fractures can also result from valgus stress to the knee and may mimic valgus instability on exam. In chronic cases of medial knee instability, longstanding cassette films should be obtained of both lower extremities to assess overall alignment [8]. MRI is useful to assess the location, grade, and other concomitant injuries to the knee such as ACL tear or medial meniscus tear.

MCL injuries are classified as grade I-tears and involve a few fibers of the MCL with localized tenderness (opening: 0-5 mm). Grade II injuries include disruption of more fibers with generalized tenderness (valgus opening: 5-10 mm). Grade III injuries are complete MCL tears with resultant medial joint laxity to valgus stress $(>10 \mathrm{~mm}$ opening).

\section{Acute isolated MCL injuries}

Grade I and II injuries or incomplete tears as described above are treated with non-operative management [9]. Treatment is directed initially with rest, cryotherapy, compression, and elevation in the acute phase $(72 \mathrm{~h})$. The initial goal is to reduce pain and swelling. The patient is 
allowed to bear weight as tolerated depending on their pain level with use of a hinged knee brace to protect the knee from further valgus stresses. Active range of motion is initiated early to prevent stiffness with concomitant strengthening exercises. Finally, once a patient's strength and proprioception have recovered to comparable levels to the other side, the patient may return to sport. Derscheid et al. [10] reported a mean return to football within 20 days in patients with grade I or II sprains who were treated nonoperatively.

Treatment of grade III or complete isolated MCL tears is more controversial than treatment of grade I or II injuries. There is evidence to support both non-operative and operative treatment of these injuries. Initially, operative treatment was advocated primarily due to the poor initial results of non-operative treatment [9]. Subsequently, however, in comparison studies no subjective or objective differences were found between surgically and non-surgically managed groups, and the pendulum has swung toward non-operative management of these isolated injuries [11, 12]. However, in a subset of patients with tibial-sided avulsions (Fig. 4) or bony avulsions, acute repair is indicated [13]. One must be careful to rule out other ligamentous injury to the knee in adults and also physeal injury in children to ensure that it is indeed an isolated MCL injury.

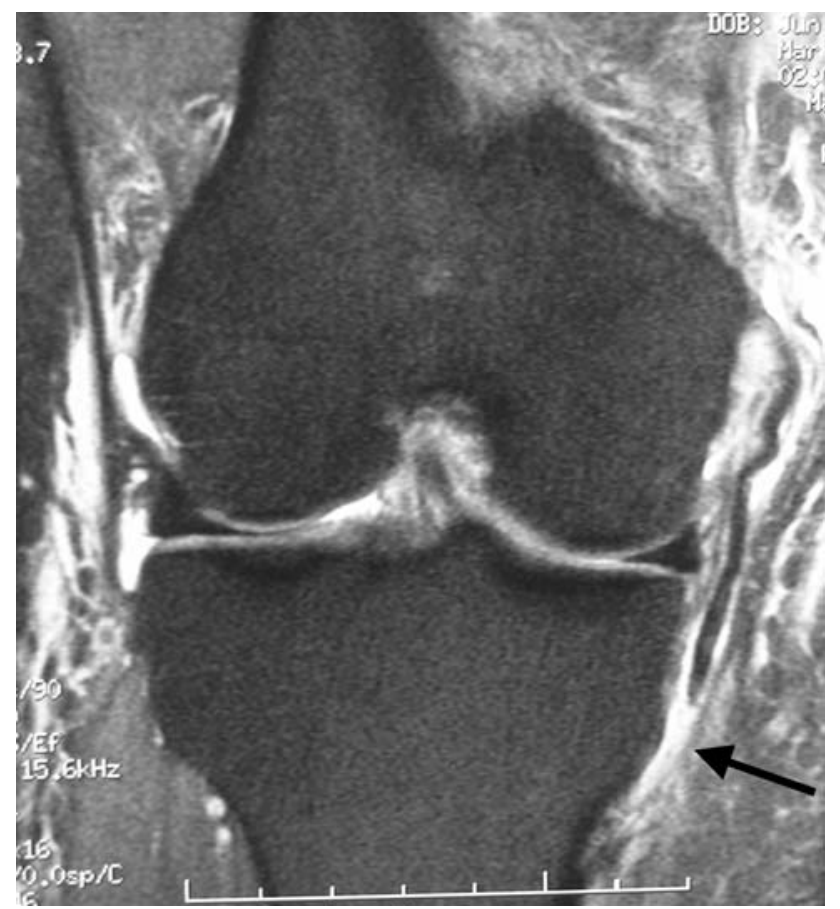

Fig. 4 Coronal MRI demonstrating complete grade 3 MCL tear from the tibial origin (black arrow)

\section{Combined acute MCL and ACL injuries}

Combined grade I or II MCL and ACL injuries are treated with non-operative treatment of the MCL with subsequent ACL reconstruction when acceptable knee motion has been attained. The goal of treatment of these combined injuries is to avoid knee stiffness; delayed reconstruction of the ACL reduces the incidence of this complication $[14,15]$.

In combined grade III MCL and ACL injuries, the MCL is still usually treated non-operatively with subsequent ACL reconstruction. In these patients, the knee is braced for 6 weeks with subsequent motion and strength exercises after the MCL is allowed to adequately heal. If persistent valgus instability remains after 6 weeks of bracing, surgical treatment for both the MCL in conjunction with ACL reconstruction is usually recommended. One recent report, however, supports non-operative management of grade III MCL tears in combination with ACL reconstruction [16]. After ACL-MCL reconstruction, the patients are made non-weight bearing and locked in extension with ROM initiated at 3 weeks post-operatively [17].

\section{Chronic MCL injuries}

Chronic valgus instability results when grade III MCL injuries fail to heal. Distal avulsions can lead to entrapment of the MCL into the joint or displacement of the distal edge over the insertion of the pes anserinus, thereby preventing healing [18]. Chronic medial instability can also result from failed ACL/PCL, or combined ligament reconstructions. Knee instability often occurs in multiple planes with rotational and translational instability in addition to valgus instability. Indications for operative reconstruction include disability that affects activities of daily living and inability to participate in athletic activities.

Patients with incompetent cruciate ligaments undergo arthroscopic cruciate reconstruction. The knee is then examined for medial laxity at 0 and $30^{\circ}$ of flexion. If there is increased laxity at $30^{\circ}$ of flexion, medial collateral ligament reconstruction is indicated. In chronic cases, every effort is made to primarily repair the MCL; however, the torn edges are often not easily identified secondary to scarring and incomplete healing. In these cases, allograft tendon (Achilles or tibialis anterior) or semitendinosus autograft has been used. More recent reports have presented semitendinosus tenodesis as a method of restoring medial instability [19]. Short-term outcome studies have shown good results with medial stability and range of motion with the use of autogenous hamstring tendons for MCL reconstruction [20]; however, the most common complication after MCL reconstruction is knee stiffness. 
Severe valgus laxity usually requires tightening of the MCL and posterior capsule including the POL [8].

Postoperative rehabilitation follows the regimen that is used for cruciate reconstruction with emphasis on achieving full extension of the knee. If only medial repair is done, the knee is braced in extension for 2-4 weeks to allow soft tissue healing. Patients then begin a range of motion exercises with strengthening beginning at 6 weeks. This includes bicycling and low-impact closed chain activities. The brace is removed at 6-8 weeks.

\section{Role of bracing in contact sports}

Numerous studies have investigated the role of prophylactic knee bracing in contact sports as well as rule modifications and promotion of fair play in preventing soft tissue knee injuries. Most have shown limited and unclear benefits and the issue is controversial [21]. There is evidence that unbraced linemen, linebackers/tight end group are more susceptible to MCL injury during competitive play [22]. Two large epidemiological studies have demonstrated consistent trends of decreased risk of MCL injury in braced football players [22-24]. Biomechanical studies also suggest that rigid knee braces can provide up to $20-30 \%$ greater MCL resistance to valgus stress [21].

The decreased risk of injury is countered by the fact that knee braces may restrict athletic performance by increasing energy expenditure, restricting motion, speed, and agility. Rigid braces can further hinder rotation, the degree of flexion as well as adduction and abduction. Styf et al. [25] studied the effect of functional bracing and intramuscular pressure and found that external compression from a knee brace causes decreased perfusion and premature muscle fatigue. Another practical concern is the difficulty in keeping a knee brace in position so that they provide protective function. In addition, differences in the effectiveness of custom-fit and off-the-shelf braces are still unclear and need further clarification.

\section{MCL injuries in children}

Ligamentous injuries of the knee in children have been historically rare $[26,27]$. The joint capsule is stronger than the physis and complete tear of the periarticular ligaments usually occurs after growth plate arrest [27]. When trauma occurs in an area with bone, ligaments, and the cartilaginous physis, the injury usually occurs at the physis [28]. With increased competitive athletics at a young age, more children are beginning to have not only physeal injuries but also ligamentous tears [29]. Fractures that involve the physis account for $20 \%$ of all childhood fractures with $3 \%$ of these occurring around the knee. Past review articles of epiphyseal injuries around the knee either do not mention ligamentous integrity or state that they are intact. More recent literature conveys that physeal fractures do not preclude ligamentous injuries and can in fact be associated with a high incidence of ligament injury [28-31].

Both ACL and MCL injuries have been reported with epiphyseal knee injuries. MCL tears usually occur in the superficial portion. The mechanism of injury begins with a valgus stress on an immature knee joint. The lateral proximal tibial physis suffers a Salter-Harris type V crushing injury. The superficial medial collateral ligament is stretched and ultimately tears. As further valgus force is applied the deep MCL remains intact, but an SH III fracture occurs through the tibial physis which can propagate into the lateral joint compartment [28]. Treatment for this injury includes reduction and fixation of the Salter-Harris III fracture and primary repair of the MCL. The patient is then followed closely for physeal arrest to prevent genu valgum deformity.

Combined ACL/MCL injuries have also been recently reported. Despite significant data on this combined injury in adults, few reports are available in the pediatric population. Sankar et al. [30] reported on a small series of pediatric ACL/MCL injuries treated with ACL reconstruction and non-operative management of MCL. At 5 years follow-up, all patients had stable knee joints and all were able to return to their pre-injury athletic level. They concluded that similar to adult injuries, bracing of grade 2 or $3 \mathrm{MCL}$ injuries followed by ACL reconstruction was an effective method of treatment for pediatric combined ACL/ MCL injuries.

\section{Summary}

The medial collateral ligament and medial-sided knee injuries are commonly encountered problems of modern sports medicine. With advances in imaging and a thorough history and physical examination, accurate characterization of each aspect of the injury is possible. Grade I and II MCL injuries can be successfully treated non-operatively with an appropriate functional rehabilitation program. Grade III injuries are more controversial, however, but most can be treated non-operatively. Combined MCL and ACL injuries are treated with non-operative treatment of the MCL with subsequent ACL reconstruction completed when normal knee motion has been attained. Chronic valgus instability from failed healing of grade III MCL tears can result in degenerative arthritis and are therefore treated operatively with MCL reconstruction. Epidemiological studies suggest prophylactic brace wear may provide some protection from medial-sided knee injury; however, they can also hinder 
athletic performance. There is currently no consensus on the use of knee bracing in preventing MCL knee injuries.

Children with medial sided knee pain should have stress radiographs of the knee to identify common physeal injuries. However, with increased intensity of competitive athletics in children, some studies have shown that epiphyseal injuries are frequently associated with ligamentous injuries. The few studies that examine these injuries in children recommend a similar treatment algorithm as in adults, with the additional concerns of following the patients for physeal arrest and genu valgus deformity.

\section{References}

1. Indelicato PA. Isolated medial collateral ligament injuries in the knee. J Am Acad Orthop Surg 1995;3(1):9-14.

2. Warren LF, Marshall JL. The supporting structures and layers on the medial side of the knee: an anatomical analysis. J Bone Joint Surg Am Vol 1979;61(1):56-62.

3. Gardiner JC, Weiss JA, Rosenberg TD. Strain in the human medial collateral ligament during valgus loading of the knee. Clin Orthop Relat Res 2001;391:266-74.

4. Grood ES, et al. Ligamentous and capsular restraints preventing straight medial and lateral laxity in intact human cadaver knees. J Bone Joint Surg Am 1981;63(8):1257-69.

5. Phisitkul P, et al. MCL injuries of the knee: current concepts review. Iowa Orthop J 2006;26:77-90.

6. Hughston JC, et al. Classification of knee ligament instabilities. Part I. The medial compartment and cruciate ligaments. J Bone Joint Surg Am 1976;58(2):159-72.

7. Zionts LE. Fractures around the knee in children. J Am Acad Orthop Surg 2002;10(5):345-55.

8. Groff Y, Harner CD. Medial collateral ligament reconstruction, In: J. DW, editor. Master techniques in orthopaedic surgery: reconstructive knee surgery. Philadelphia: Lippincott Williams \& Wilkins; 2003.

9. Kannus P. Long-term results of conservatively treated medial collateral ligament injuries of the knee joint. Clin Orthop Relat Res 1988;226:103-12.

10. Derscheid GL, Garrick JG. Medial collateral ligament injuries in football. Nonoperative management of grade I and grade II sprains. Am J Sports Med 1981;9(6):365-8.

11. Indelicato PA. Non-operative treatment of complete tears of the medial collateral ligament of the knee. J Bone Joint Surg Am 1983;65(3):323-9.

12. Reider B, et al. Treatment of isolated medial collateral ligament injuries in athletes with early functional rehabilitation. A fiveyear follow-up study. Am J Sports Med 1994;22(4):470-7.
13. Wilson TC, Satterfield WH, Johnson DL. Medial collateral ligament "tibial" injuries: indication for acute repair. Orthopedics 2004;27(4):389-93.

14. Noyes FR, Mangine RE, Barber SD. The early treatment of motion complications after reconstruction of the anterior cruciate ligament. Clin Orthop Relat Res 1992;277:217-28.

15. Petersen $\mathrm{W}$, Laprell $\mathrm{H}$. Combined injuries of the medial collateral ligament and the anterior cruciate ligament. Early ACL reconstruction versus late ACL reconstruction. Arch Orthop Trauma Surg 1999;119(5-6):258-62.

16. Halinen J, et al. Operative and nonoperative treatments of medial collateral ligament rupture with early anterior cruciate ligament reconstruction: a prospective randomized study. Am J Sports Med 2006;34(7):1134-40.

17. Edson CJ. Conservative and postoperative rehabilitation of isolated and combined injuries of the medial collateral ligament. Sports Med Arthrosc 2006;14(2):105-10.

18. Robins AJ, Newman AP, Burks RT. Postoperative return of motion in anterior cruciate ligament and medial collateral ligament injuries. The effect of medial collateral ligament rupture location. Am J Sports Med 1993;21(1):20-5.

19. Kim SJ, Choi NH, Shin SJ. Semitendinosus tenodesis for medial instability of the knee. Arthroscopy 2001;17(6):660-3.

20. Yoshiya $\mathrm{S}$, et al. Medial collateral ligament reconstruction using autogenous hamstring tendons: technique and results in initial cases. Am J Sports Med 2005;33(9):1380-5.

21. Najibi S, Albright JP. The use of knee braces, part 1: prophylactic knee braces in contact sports. Am J Sports Med 2005;33(4):602-11.

22. Albright JP, et al. Medial collateral ligament knee sprains in college football. Effectiveness of preventive braces. Am J Sports Med 1994;22(1):12-8.

23. Sitler $M$, et al. The efficacy of a prophylactic knee brace to reduce knee injuries in football. A prospective, randomized study at West Point. Am J Sports Med 1990;18(3):310-5.

24. Albright JP, et al. Medial collateral ligament knee sprains in college football. Brace wear preferences and injury risk. Am J Sports Med 1994;22(1):2-11.

25. Styf JR, Nakhostine M, Gershuni DH. Functional knee braces increase intramuscular pressures in the anterior compartment of the leg. Am J Sports Med 1992;20(1):46-9.

26. Salter RB. Injuries of the epiphyseal plate. Instr Course Lect 1992;41:351-9.

27. Salter RB, Harris WR. Injuries involving the epiphyseal plate. J Bone Joint Surg Am 1963;45-A:587-622.

28. Bertin KC, Goble EM. Ligament injuries associated with physeal fractures about the knee. Clin Orthop Relat Res 1983;177:188-95.

29. DeLee JC, Curtis R. Anterior cruciate ligament insufficiency in children. Clin Orthop Relat Res 1983;172:112-8.

30. Sankar WN, et al. Combined anterior cruciate ligament and medial collateral ligament injuries in adolescents. J Pediatr Orthop 2006;26(6):733-6.

31. Steadman JR. Rehabilitation of first- and second-degree sprains of the medial collateral ligament. Am J Sports Med 1979;7(5):300-2. 